\title{
Variación estacional de lípidos en varios tejidos del cambute Strombus gigas (Mesogastropoda: Strombidae), en Quintana Roo, México
}

\author{
Nancy Brito Manzano y Dalila Aldana Aranda ${ }^{1}$ \\ 1 CINVESTAV IPN Unidad Mérida, Laboratorio de Biología Marina, Carretera Antigua a Progreso km 6, C.P. 97310, Mérida, \\ Yucatán, México. Fax (99) 81-29-05, E-mail: nbrito@kin.cieamer.conacyt.mx
}

\author{
Recibido 19-I-1998. Corregido 15-IV-1998. Aceptado 29-IV-1998.
}

\begin{abstract}
Strombus gigas samples were collected in Banco Chinchorro, Quintana Roo, Mexico $\left(18^{\circ} 47^{\prime}-18^{\circ} 23^{\prime}\right.$ N; $\left.87^{\circ} 14^{\prime} \mathrm{W}\right)$. Shell, body and spire lenght were measured. Fat content of gonad, digestive gland and meat of male and female conchs, was analyzed by the standard and microsoxhlet methods on a monthly basis for a year. Mean lenght for shell, body and spire were $209 \pm 1 \mathrm{~mm}, 137 \pm 1 \mathrm{~mm}$ and $100 \pm 1 \mathrm{~mm}$, respectively. Total wet weigth was $1826 \pm 7 \mathrm{~g}$. Fat content in gonad-digestive gland was $1.27-9.33 \mathrm{~g} \%$ with a mean of $4.27 \mathrm{~g} \%$ and in meat $0.41-5.03 \mathrm{~g} \%$ with a mean of $2.15 \mathrm{~g} \%$. Fat content in female gonad-digestive gland was $1.27 \mathrm{~g} \%$ at $8.57 \mathrm{~g} \%$ with a mean of $4.50 \mathrm{~g} \%$; in male: 1.87 $\mathrm{g} \%$ at $9.33 \mathrm{~g} \%$ with a mean of $4.01 \mathrm{~g} \%$. Sexes differed (t student $\mathrm{P}<0.05$ ). Mean fat content in meat was $2.15 \mathrm{~g} \%$, (males $1.97 \mathrm{~g} \%$, females $2.3 \mathrm{~g} \%$ ). There was not significant sexual difference-for meat fat content. There was a difference between gonad-digestive gland and meat ( $t$ student $P<0.05$ ). However, from February to August meat lipid content exceeds the percentage recommended for human consumption by the FAO.
\end{abstract}

Key words: Strombus, lipid composition, annual cycle.

El cambute $S$. gigas ha sido por generaciones, una fuente primordial de proteínas para los habitantes del Caribe, su concha es utilizada con propósitos ornamentales y artesanales y las vísceras se emplean como cebo para la pesca (Brownell y Stevely 1981) o se cuecen para elaborar embutidos, como es el caso en las antillas francesas (Rathier 1993). Appeldoorn (1994), estimó para 1991 una captura anual de 1500 a 4000 toneladas de carne de cambute proveniente de los diferentes países del Caribe, lo que representó un valor comercial en el mercado de 30 millones de dólares. Una de las razones por la que la producción de cambute ha disminuído, es debido a la intensificación de la explotación en el Caribe. Esta se acentúa a partir de 1970, en que aumenta la demanda del producto por Estados Unidos y por el mercado europeo en 1982. Ambos mercados internacionales presentan una demanda de cambute no satisfecha. Otra de las razones por la cual la producción de este gasterópodo ha declinado, es por la modificación ecológica del hábitat donde regularmente se desarrolla, lo que ha disminuído aún más, las ya escasas poblaciones existentes, colocando a la especie en una situación crítica para su supervivencia (Rathier 1993). Por último se trata de un recurso comunitario del Caribe, por lo que las 
medidas biológico-pesqueras para su regulación tendrían que ser tomadas y aplicadas de manera inter-países en esta región (Stoner 1996).

Hoy en día se busca para las dietas modernas alimentos magros; así, en la medida que la carne de cambute se consume todo el año y dependiendo de la tradición culinaria de cada uno de los países del Caribe, se consumen diferentes tejidos, se determinó realizar para el presente estudio un análisis del contenido de lípidos en gónada-glándula digestiva y en la parte comestible del cambute (músculo) a lo largo de un ciclo anual. Además en este estudio se separaron para su análisis de contenido de lípidos las hembras y los machos, en la medida que las hembras almacenan lípidos sobre todo en glándula digestiva en el período pre-vitelogenético y en gónada en el de vitelogenesis (Voogt 1980).

En este trabajo se realiza por primera vez un análisis del contenido de lípidos a lo largo de todo un ciclo anual, por sexos separados y para diferentes tejidos del cambute, a diferencia de otros estudios donde los análisis han sido puntuales con un número reducido de animales sin tomar en cuenta el sexo, ni las variaciones estacionales. Además ellos han sido realizados solo para el músculo.

\section{MATERIALES Y METODOS}

Se capturaron por medio de buceo autónomo 36 organismos mensualmente a lo largo de un año en Banco Chinchorro, México. Banco Chinchorro está ubicado a $18^{\circ} 47^{\prime}-18^{\circ} 23^{\prime} \mathrm{N}$ y $87^{\circ} 14^{\prime} \mathrm{E}$, es un arrecife coralino que se encuentra a $30 \mathrm{~km}$ de la costa sureste del Estado de Quintana Roo, México. Mide $40 \mathrm{~km}$ de largo y $18 \mathrm{~km}$ de ancho, abarca $700 \mathrm{~km}^{2}$ de superficie, siendo el arrecife más grande de todo el Caribe (Aguilar Perera 1992).

De cada uno de los cambutes se registró el peso húmedo total, para lo cual se utilizó una balanza de plato con una precisión de $\pm 25 \mathrm{~g}$. La longitud de la heliconcha, del cuerpo y de la espira se midieron con una regla de $30 \mathrm{~cm}$ con divisiones de un $\mathrm{mm}$, registrando los valores promedio y su desviación estándar. Posteriormente se procedió a separar la carne de la concha de cada cambute y en uná balanza con precisión de $0.01 \mathrm{~g}$ se registró el peso de los tejidos blandos. El cuerpo se disectó, separando la masa visceral y gónada de los músculos de la columela y del pie (parte comestible), éstas partes se pesaron por separado. Para cada organismo se registró el sexo del animal.

Para el análisis de lípidos se tomaron 138 organismos, mensualmente fueron analizados seis organismos hembras y seis organismos machos para los tejidos de la parte comestible y gónadaglándula digestiva. Las muestras de gónadaglándula digestiva, que en esta especie se encuentran formando un tejido interconectado, se tomaron en su parte media. Las muestras correspondientes a la parte comestible, fueron tomadas del músculo en la sección contigua al opérculo.

Las muestras de estos tejidos disectados, se pesaron en una balanza con precisión de $0.01 \mathrm{~g}$, posteriormente fueron desecadas a $60^{\circ} \mathrm{C}$ en una estufa durante $72 \mathrm{hr}$, que es el tiempo requerido por las muestras para obtener un valor de humedad constante (Anónimo 1984).

Para el análisis de lípidos se seleccionaron tres muestras de cada uno de los tejidos estudiados, incluyendo organismos de ambos sexos. Las muestras se molieron teniendo cuidado de no perder alguna fracción de la muestra. El peso de la muestra seca molida en todos los casos fue superior a dos g, que es la cantidad mínima requerida para efectuar el análisis de acuerdo a las técnicas establecidas por Anónimo (1984). Para la determinación de lípidos crudos se utilizó el método Microsoxhlet, la cual consiste en poner una muestra seca en éter etílico anhidro en un dedal de papel filtro que permite el paso rápido del disolvente. El tiempo de extracción puede variar desde cuatro hr a velocidad de condensación de cinco a seis gotas por s, hasta 16 hr de dos a tres gotas por s. Recuperar el éter y evaporar el residuo sobre baño maría en lugar bien ventilado, secar el residuo a $100^{\circ} \mathrm{C}$ durante $30 \mathrm{~min}$, enfriar y pesar hasta peso constante. El peso de lípidos crudos en la muestra es obtenido de la diferencia entre el peso del matraz limpio y seco y el peso del matraz con el residuo de 
grasas. El resultado es expresado como $\mathrm{g} \%$ del peso de la muestra inicial.

\section{RESULTADOS}

El peso húmedo total promedio de los organismos fue de $1826 \pm 7 \mathrm{~g}$. Los valores promedios de las mediciones realizadas sobre las longitudes de la heliconcha, de la espira y el cuerpo de los organismos, fueron de $209 \pm 1 \mathrm{~mm}$ ( \pm 1 D.E.), $100 \pm$ $1 \mathrm{~mm}$ y $1371 \mathrm{~mm}$, respectivamente.

En la Fig. 1 A se presentan los valores del contenido de lípidos en base seca para hembras y machos en gónada-glándula digestiva. El valor máximo para las hembras se obtuvo en junio con $8.57 \mathrm{~g} \%$ y el valor mínimo en diciembre con $1.27 \mathrm{~g} \%$, el valor medio fue de $4.50 \pm 0.03 \mathrm{~g} \%$. Para los machos los valores máximo y mínimo se presentaron en agosto y diciembre con $9.33 \mathrm{~g} \%$ y $1.87 \mathrm{~g} \%$ respectivamente, el valor medio fue de $4.01 \pm 0.01 \mathrm{~g} \%$. De agosto a febrero se encontraron más lípidos en machos con respecto a las hembras, $(\mathbf{t}$ de student $\mathbf{p}<0.05)$, con excepción de noviembre. Por el contrario, de marzo a junio se encontraron más lípidos en hembras que en machos, ( $t$ de student $p<0.05$ ). Al realizar la comparación del contenido de lípidos en la gónada-glándula digestiva entre las hembras del cambute, se encontró que éstas presentaron diferencias significativas de febrero a agosto. Las diferencias no fueron significativas de septiembre a enero. Para los machos, solo hubo diferencias significativas en agosto ( $t$ de student $\mathrm{p}<0.05$ ).

En la Fig. 1 B se observa el contenido de lípidos en base seca para hembras y machos en la parte comestible del cambute. El valor máximo en las hembras se presentó en junio con $5.03 \mathrm{~g} \%$ y el mínimo con $0.55 \mathrm{~g} \%$ en mayo, mientras que para los machos el valor máximo se obtuvo en febrero con $4.86 \mathrm{~g} \%$ y el valor mínimo se presentó en mayo con $0.41 \mathrm{~g} \%$. Los valores de lípidos de la parte comestible del cambute no mostraron diferencia sexual ( $t$ de student $p<0.05$ ). Al realizar la comparación del contenido de lípidos en la parte comestible entre las hembras se encontró que éstas si presentaron diferencias significativas de febrero a agosto, y que las diferencias no fueron significativas de septiembre a enero. Para los machos, las diferencias fueron significativas de febrero a agosto ( $t$ de student $\mathrm{p}<0.05)$.

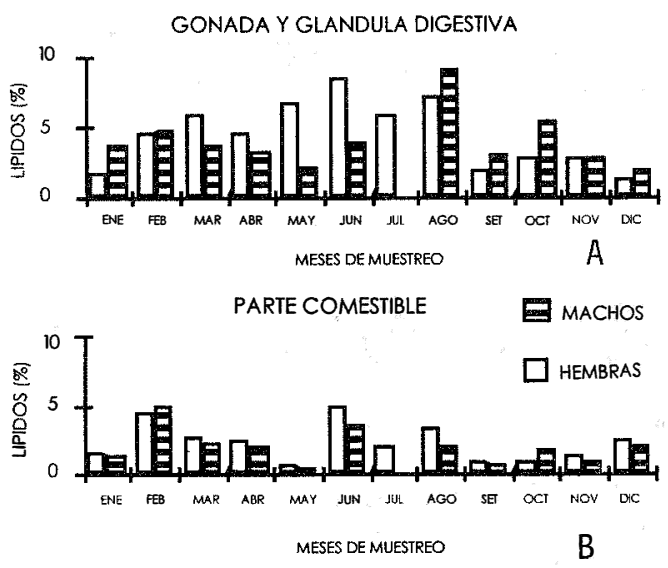

Fig. 1. Contenido de lípidos en base seca en gónada-glándula digestiva (A) y parte comestible (B) a lo largo del ciclo anual para hembras y machos del cambute Strombus gigas, del arrecife Banco Chinchorro, Quintana Roo, México ( $n=12$ organismos analizados mensualmente).

\section{DISCUSION}

Los estudios realizados sobre la composición de lípidos con diferentes especies de cambutes siempre han sido realizados con muestras puntuales y solo para el tejido de la parte comestible, como lo reportado en los trabajos realizados con S. galeatus por Blanco Metzler y Montero Campos (1992), con S. costatus por González Ferráez (1989) y Morales Adrián (1990). En estos estudios no se considera ni el sexo del molusco, ni las variaciones eventuales que puedan presentarse a lo largo de un ciclo anual en los tejidos del animal, ni las variaciones inter-tejidos.

En el presente trabajo se realizó la determinación de lípidos, que actualmente es un tema de interés en la nutrición humana para el consumo de alimentos marinos con bajo contenido de 
grasas. Se analizó la evolución de lípidos en gónada-glándula digestiva y músculo del pie a lo largo de un ciclo annual, realizándose éstos por separado para hembras y machos, a fin de conocer el comportamiento de los lípidos en la carne del cambute que es la principal parte comestible, pero también en la gónada-glándula digestiva. Lo anterior debido a que en algunos países del Caribe por sus preferencias culturales y culinarias, se consume el animal completo para la elaboración de embutidos. González Ferráez (1982), Morales Adrián (1990), Blanco Metzler y Montero Campos (1992) encontraron valores bajos del contenido de lípidos en la parte comestible del cambute por lo que reportan que es bueno para consumo humano, sin embargo

\section{CUADRO 1}

Comparación del contenido de lípidos de algunos organismos marinos con los de la carne de diferentes especies de cambute. Los datos de los autores Morales Adrián y Blanco Metzler y Montero Campos son puntuales. Los datos para Strombus gigas en este estudio son el valor medio obtenido a lo largo de un ciclo anual.

Organismo

Camarón

Calamar

Pescado Mero

Pulpo

Langostino

Strombus costatus

Strombus galeatus

Strombus gigas

* Reportados en base húmeda

** Reportado en base seca
Porcentaje de lípidos

$0.2^{*}$
$0.9^{*}$
$0.14^{*}$
$0.32^{*}$
$4.26^{*}$
$2.78^{* *}$
$0.26^{*}$

0.11 a $1.35^{*}$

\section{Fuente}

Adams (1975)

Adams (1975)

Adams (1975)

Adams (1975)

Adams (1975)

Morales Adrián (1990)

Blanco Metzler y Montero Campos (1992)

Este estudio

\section{CUADRO 2}

Datos de la literatura sobre el porcentaje de lípidos en la parte comestible de diferentes gasterópodos marinos y datos de este estudio para la parte comestible y gónada-glándula digestiva de Strombus gigas durante un ciclo anual. Entre paréntesis valor promedio. Valores reportados en base húmeda.

Gasterópodos

Busycon carica

Pleuroploca gigantea

Xancus angulatus

Strombus costatus

Strombus galeatus

Strombus gigas (Músculo)

Strombus gigas

(Músculo de hembras)

Strombus gigas

(Músculo de machos)

Strombus gigas

(Gónada-Glándula digestiva)

Strombus gigas

(Gónada-Glándula digestiva de hembras)

Strombus gigas

(Gónada-Glándula digestiva de machos)
Porcentaje de lípidos

0.09

0.27

0.06

0.83

0.26

0.41 a $5.03(2.15)$

0.55 a $5.03(2.3)$

0.41 a $4.86(1.97)$

1.27 a $9.33(4.27)$

1.27 a $8.57(4.50)$

1.87 a $9.33(4.01)$
Fuente

Morales Adrián (1990)

Morales Adrián (1990)

Morales Adrián (1990)

Morales Adrián (1990)

Blanco Metzler y Montero Campos (1992)

Este estudio

Este estudio

Este estudio

Este estudio

Este estudio

Este estudio 
realizaron un análisis puntual, para un sólo tejido y no tomaron en cuenta el sexo del animal ni la época del año. En el presente trabajo se encontraron valores bajos del contenido de lípidos en el músculo del cambute y en general resulta una carne magra óptima para consumo humano. Sin embargo dependiendo de la época del año, existen diferencias, siendo la carne más magra para el período de septiembre a enero ( $2 \mathrm{~g} \%$ ) contrariamente de febrero a agosto el contenido es de casi el doble (3.6 g\%).

En gónada-glándula digestiva el contenido de lípidos es más elevado con respecto al músculo y además durante el período de febrero a agosto se presenta el valor máximo de casi 10 , mientras que de septiembre a enero el contenido es en promedio de $3 \mathrm{~g} \%$. En hembras de febrero a agosto el contenido de lípidos fue mayor con respecto al de machos. Los resultados anteriores repercuten en la dieta para consumo humano, ya que la ingesta de lípidos va a ser mayor si se consumen organismos hembras.

Estudios puntuales efectuados con otros organismos marinos como pescados frescos, crustáceos y moluscos presentan cantidades de grasa similares o mayores a las que se obtuvieron en el presente estudio, como se muestra en el Cuadro 1. En el Cuadro 2 se presenta una comparación entre el porcentaje de lípidos determinado en este estudio y lo reportado por otros autores para diferentes especies de gasterópodos.

De esta manera se observó que no es lo mismo consumir la parte comestible del cambute, que la gónada-glándula digestiva, ya que ésta presenta un contenido de lípidos mayor. De igual manera, no es lo mismo el consumo de estos dos tejidos a lo largo de un ciclo anual, en virtud de que existen meses durante los cuales se presenta un mayor contenido de lípidos, que puede ser hasta diez veces más alto. Por lo que respecta a los valores del contenido de lípidos en la parte comestible, estos indican que dependiendo de la época del año, se puede comer el doble o el triple de grasa.

Nuestros resultados para la parte comestible y gónada-glándula digestiva del cambute $S$. gigas, muestran que existen variaciones estacionales, inter-tejidos y ligados al sexo. Por lo que se recomienda su consumo de preferencia de septiembre a enero, donde la ingesta de lípidos es tres veces menor.

\section{AGRADECIMIENTOS}

Agradecemos a la CEE y al Consejo Científico y Técnico del Gobierno de Francia el apoyo económico brindado, a Manuel Sánchez Crespo y a los pescadores de Banco Chinchorro en la obtención de organismos, y los laboratorios de biología marina y nutrición acuícola del CINVESTAV IPN, donde se realizó la preparación de muestras y determinación de lípidos, respectivamente.

\section{RESUMEN}

Se determinó la variación mensual de lípidos en varios tejidos del cambute Strombus gigas (L.), en Banco Chinchorro, Quintana Roo, México durante un año. La longitud de la heliconcha fue $209 \pm 1 \mathrm{~mm}$, la del cuerpo $137 \pm 1$ $\mathrm{mm}$, la de la espira $100 \pm 1 \mathrm{~mm}$ y el peso húmedo total 1 $826 \pm 7 \mathrm{~g}$. La media del contenido de lípidos en la gónada-glándula digestiva fue $4.27 \mathrm{~g} \%$ (machos $4.01 \mathrm{~g} \%$ y hembras $4.50 \mathrm{~g} \%$ ). El contenido de lípidos en gónadaglándula digestiva mostró diferencias sexuales significativas $(\mathrm{p}<0.05)$. En la porción comestible la media fue 2.15 $\mathrm{g} \%$, (machos $1.97 \mathrm{~g} \%$, hembras $2.3 \mathrm{~g} \%$ ). Las diferencias sexuales no fueron significativas ( $t$ de student $p<0.05$ ). El análisis estadístico mostró diferencias significativas ( $\mathrm{p}<$ 0.05 ), entre los valores obtenidos en gónada-glándula digestiva con respecto a la parte comestible. Sin embargo, de febrero a agosto el contenido de lípidos en la parte comestible excede el porcentaje de lípidos para el consumo humano recomendado por la FAO.

\section{REFERENCIAS}

Adams, C.F. 1975. Nutritive value of American foods in Common Units. Agriculture Handbook No. 456. USDA, Washington, D.C. 253 p.

Aguilar Perera, R. 1992. Banco Chinchorro. Departamento de Acuacultura y pesquerías. CIQROO. 2 p. Diario de Yucatán (22 octubre).

Anónimo 1984. Association of Official Analytical Chemists. Official Methods of Analysis of the AOAC. Washington, D.C. 1141 p. 
Appeldoorn, R.S. 1994. Queen conch management and research: status, needs and priorities, p. 301-319. In R.S. Appeldoorn \& B. Rodríguez (eds.). Biología, pesquería y cultivo del caracol Strombus gigas. Ex libris, Caracas.

Blanco Metzler, A. y M. Montero Campos. 1992. Composición químico-nutricional de la carne de cambute, Strombus galeatus (Mesogastropoda: Strombidae). Rev. Biol. Trop. 40: 89-93.

Brownell, W.N. \& J.M. Stevely. 1981. The biology, fisheries and management of the queen conch, Strombus gigas. Mar. Fish. Rev. 43: 1-2.

González Ferráez, J.A. 1989. Análisis Bromatológico y determinación cualitativa de aminoácidos del caracol Strombus gigas. Tesis de Licenciatura, Instituto Tecnológico de Mérida, México.

Morales Adrián, M. 1990. Aporte nutricional del caracol blanco, Strombus costatus (Gmelin). Tesis de
Licenciatura, Universidad Autónoma de Yucatán, Mérida, México.

Rathier, Y. 1993. Le stock de lambis (Strombus gigas, L.) en Martinique: Analyse de la situation 1986-1987, modelisation de l'exploitation, options de gestion et d'amenagement. Thèse de Doctorat, Université de Bretagrie Occidentale, Brest, Francia.

Stoner, A.W. 1996. Status of Queen conch research in the caribbean, p. 23-39. In Posadas, García-Moliner and Oliveras (eds.). International Queen Conch Conference. Caribbean Fishery Management Council. San Juan, Puerto Rico.

Voogt, P. A. 1980. Lipids: Their Distribution and Metabolism. pp: 329-360. In: K. M. Wilbur (ed.). The Mollusca. Academic, Nueva York 